\title{
A coordinate independent formulation of the Weyl-Wigner transform theory
}

\author{
Augusto César Lobo ${ }^{\mathrm{a}, *}$, Maria Carolina Nemes ${ }^{\mathrm{b}}$ \\ ${ }^{a}$ Departamento de Física, ICEB-UFOP, CEP 35400-000, Ouro Preto, MG, Brazil \\ ${ }^{\mathrm{b}}$ Departamento de Física, ICEX-UFMG, CEP 31270-010, Belo Horizonte, MG, Brazil
}

Received 20 November 2001; received in revised form 6 March 2002

\begin{abstract}
We present the Weyl-Wigner (WW) transform theory in a much more compact way than usual, by introducing the $\Delta$ basis in an intrinsic form. This permits the derivation of new identities and also leads to generalizations, like the inclusion of finite-dimensional systems in the WW theory, which is also discussed. We show, in this case, some striking differences in the structure of finite phase space depending on the underlying dimension of quantum space being an even or odd integer. (c) 2002 Elsevier Science B.V. All rights reserved.
\end{abstract}

\section{Introduction}

The Weyl-Wigner (WW) formalism introduced first by Weyl in the early years of quantum theory has become, since then, a very powerful mathematical instrument in quantum mechanics to study classical limits, for instance, as well as in other applied physical-mathematical sciences like quantum statistical physics, information processing and optics [1-4]. It enables us to establish a one-to-one relation between quantum observables (Hermitian operators) and classical observables (real functions on phase space).

These functions are just the components of the Hermitian observables in a certain special basis in operator space. This basis (the Weyl $\Delta$-operators), in turn, is formed by a very unique set of operators which is parametrized by the points of classical phase space. We introduce here an intrinsic formulation of the WW theory, in the sense that we exhibit each of these basis operators as a simple finite product of well-known quantum mechanical operators. This makes the whole theory much more compact and

\footnotetext{
* Corresponding author.

E-mail addresses: lobo@iceb.ufop.br (A.C. Lobo), carolina@sucuri.fisica.ufmg.br (M.C. Nemes).
} 
neat, so that certain generalizations, like, for instance, the extension of the WW theory to include finite-dimensional systems becomes a straightforward task.

In the next section, we use a notation different from usual so that we can describe intrinsically the Fourier transform operator which plays a central role in our "coordinate free" definition of the Weyl $\Delta$-operators. In Section 3, we state our intrinsic definition for the $\Delta$ operators and we are able to show some new mathematical identities. In Section 4 we use our approach to discuss an extension of the WW theory that includes finite-dimensional systems. We end with Section 5, where we state some conclusions and make some closing remarks. In the appendix, we prove some mathematical identities used in the paper.

\section{The Fourier transform operator}

Let $|q(x)\rangle$ and $|p(x)\rangle$ be a complete set of eigenkets of the position and momentum operators

$$
Q|q(x)\rangle=x|q(x)\rangle \text { and } P|p(x)\rangle=x|p(x)\rangle, \quad(-\infty<x<+\infty)
$$

with

$$
\int_{-\infty}^{+\infty} \mathrm{d} x|q(x)\rangle\left\langle q(x)\left|=\int_{-\infty}^{+\infty} \mathrm{d} x\right| p(x)\right\rangle\langle p(x)|=I \quad \text { (completeness) }
$$

and

$$
\langle q(x) \mid q(x)\rangle=\langle p(x) \mid p(x)\rangle=\delta(x-x) \quad \text { (normalization) }
$$

and also

$$
\langle q(x) \mid p(x)\rangle=\mathrm{e}^{\mathrm{i} x x^{\prime}} \quad(\hbar=1)(\text { Overlap }) .
$$

These continuously indexed bases can be rigorously defined in the Rigged-Hilbert Space approach to quantum mechanics [5]. Notice that we use a notation different from the usual as we distinguish the "type" of the eigenstate, $\mathbf{q}$ or $\mathbf{p}$, from its continuous $\mathfrak{R}$-valued eigenvalue $\mathbf{x}$. The symbol $q(x)$ inside the bras and kets is not to be understood as a function but as a label for a position eigenket with eigenvalue $\mathbf{x}$. That is, given any abstract physical state-vector $|\Psi\rangle$ representing the state of the non-relativistic one-dimensional motion of a single particle, its wave function in the position representation is given by $\Psi(x)=\langle q(x) \mid \Psi\rangle$. This contrasts with the usual notation $\Psi(x)=\langle x \mid \Psi\rangle$.

For example, our choice of notation permits us to write down a compact formula for the unitary Fourier transform operator that would not be possible in the usual notation

$$
F=\int_{-\infty}^{+\infty} \mathrm{d} x|p(x)\rangle\langle q(x)| .
$$

In fact, we see that

$$
F|q(x)\rangle=|p(x)\rangle \quad \text { and } \quad F|p(x)\rangle=|q(-x)\rangle .
$$


The $F^{2}$ operator is then, nothing more than a $\pi / 2$ radian rotation in phase space, so it is just the space inversion operator. We can easily see that

$$
F^{3}=F^{\dagger} \quad \text { and } \quad F^{4}=I
$$

so that the eigenvalues of $F$ are the fourth roots of unity. In fact, it is well known that (see Ref. [6] for instance)

$$
F|n\rangle=(i)^{n}|n\rangle \quad \text { where }\{|n\rangle\},(n=0,1,2 \ldots)
$$

is the complete set of eigenstates of the number operator

$$
N=a^{\dagger} a=\frac{1}{2}\left(Q^{2}+P^{2}-I\right),
$$

where, of course,

$$
a=\frac{1}{\sqrt{2}}(Q+i P)
$$

is the usual annihilation operator. Given a state $|\Psi\rangle$ the wave function associated to this vector is

$$
\langle q(x) \mid \Psi\rangle=\Psi(x)
$$

so that its Fourier transform is

$$
\langle p(x) \mid \Psi\rangle=\left\langle q(x)\left|F^{\dagger}\right| \Psi\right\rangle \text {. }
$$

The number operator is the generator of rotations in phase space. The best way to see this is within the complex coherent state representation. In fact, if we identify the phase plane with the complex plane by

$$
z=x+i y \text { and }|z\rangle=D[z]|0\rangle,
$$

where $q=x \sqrt{2}$ and $p=y \sqrt{2}$ are the canonical variables of the phase space plane and

$$
D[z]=\mathrm{e}^{\left(z a^{\dagger}-\bar{z} a\right)}
$$

is the usual Weyl Displacement operator in complex representation with the vector $|0\rangle$ as the ground state, that is

$$
N|0\rangle=0
$$

then it is not difficult to show the well-known result (see Ref. [7])

$$
\mathrm{e}^{\mathrm{i} \theta N}|z\rangle=\left|\mathrm{e}^{\mathrm{i} \theta} z\right\rangle \text {. }
$$

The $\mathrm{e}^{\mathrm{i} \theta N}$ operator is sometimes called the fractional Fourier transform [8].

\section{The intrinsic Weyl-Wigner operator}

\subsection{The translation operators}

Let $U_{\xi}$ and $V_{\eta}$ be, respectively, the unitary abelian representation of translation operators in the momentum and position spaces. Then

$$
V_{\eta}|q(x)\rangle=|q(x-\eta)\rangle \quad \text { and } \quad U_{\xi}|p(x)\rangle=|p(x+\xi)\rangle
$$


together with

$$
V_{\eta} V_{\eta^{\prime}}=V_{\eta+\eta^{\prime}} \quad \text { and } \quad U_{\xi} U_{\xi^{\prime}}=U_{\xi+\xi^{\prime}}
$$

and also

$$
V_{\eta}^{\dagger}=V_{\eta}^{-1} \quad \text { and } \quad U_{\xi}^{\dagger}=U_{\xi}^{-1} .
$$

They are well known to be generated by the momentum and position observables

$$
V_{\eta}=\mathrm{e}^{\mathrm{i} \eta P} \quad \text { and } \quad U_{\xi}=\mathrm{e}^{\mathrm{i} \xi Q} .
$$

Either from the Baker-Hausdorff-Campbell $(\mathrm{BCH})$ lemma or by acting on the position or momentum basis with these translation operators (Eq. (3.1)) one can easily derive the so-called Weyl commutation relation [9]

$$
V_{\eta} U_{\xi}=\mathrm{e}^{\mathrm{i} \xi \eta} U_{\xi} V_{\eta},
$$

and we also have the commutation relations between the translation operators with the Fourier transform and the square of the Fourier transform operator:

$$
F V_{\eta} F^{\dagger}=U_{\eta}^{\dagger} \quad \text { and } \quad F U_{\eta} F^{\dagger}=V_{\eta}
$$

from which we have

$$
F^{2} V_{\eta} F^{2}=V_{\eta}^{\dagger} \quad \text { and } \quad F^{2} U_{\eta} F^{2}=U_{\eta}^{\dagger} .
$$

The above equations are very important for our discussion in Section 3.3.

\subsection{The Dirac "bra" and "ket" notation for operator space}

The bra and ket notation of Dirac is so good in exhibiting explicitly the Hermitian linear algebraic structure of quantum vector spaces that we desire to extend it to operator space. Let us denote as $W$ the quantum space of states and $T_{1}^{1}(W)$ (the $\left(\begin{array}{l}1 \\ 1\end{array}\right)$ type tensors defined on $W$ ) for the space of linear operators acting on $W$. We may introduce an Hermitian inner product in $T_{1}^{1}(W)$ in the following form [10]. Let the elements $A, B, C, \ldots$ of $T_{1}^{1}(W)$ (which of course is also a vector space) be written as $\mid A), \mid B), \mid C), \ldots$ so that we can define the Hermitian inner product as

$$
(A \mid B)=\operatorname{tr}\left(A^{\dagger} B\right) \text {. }
$$

The $T_{1}^{1}(W)$ space has an additional algebraic structure which makes it an operator algebra. In fact, we have the product

$$
\mid A) \cdot \mid B)=\mid A B),
$$

where $A B$ is the usual operator product of $A$ and $B$. Note that the "dual" of an element $A=\mid A)$ is the adjoint operator $A^{\dagger}=(A \mid$. We can, in this way, identify the elements of $T_{1}^{1}(W)$ with the elements of $T_{1}^{1}\left(T_{1}^{1}(W)\right)$, the linear operators that act upon $T_{1}^{1}(W)$, in a canonical manner by the following linear injective inclusion map:

$$
\begin{aligned}
& i: T_{1}^{1}(W) \rightarrow T_{1}^{1}\left(T_{1}^{1}(W)\right) \\
& A \mapsto \hat{A}
\end{aligned}
$$


such that

$$
\stackrel{\cap}{A} \mid B)=\mid A B)
$$

We will usually not mention explicitly the inclusion map. For instance, we may write the identity operator $I$ in any one of the three forms below

$$
I \equiv \mid I) \equiv \stackrel{\cap}{I} \text {. }
$$

Let $\{X(\alpha)\}$ be a set of operators indexed by a continuous-valued parameter $\alpha$

$$
X(\alpha) \equiv \mid X(\alpha))
$$

We say that this set is complete if

$$
\left.\int \mathrm{d} \mu(\alpha) \mid X(\alpha)\right)(X(\alpha)|=I=| I)=\stackrel{\cap}{I},
$$

where $\mathrm{d} \mu(\alpha)$ is an appropriate measure and we say that the set is orthonormal if

$$
(X(\alpha) \mid X(\beta))=\operatorname{tr}\left(X^{\dagger}(\alpha) X(\beta)\right)=\delta(\alpha-\beta) .
$$

For instance, consider the family of operators $|q(x)\rangle\langle p(y)|$, which we shall denote by $\mid x, y)$. Then we can prove that this set is "complete" and "orthonormal" in operator space.

Note here that $\alpha$ is a point of $\mathfrak{R}^{2}$. In fact, it is straightforward to show that (using Eq. (3.8)

$$
\left(x, y \mid x^{\prime}, y^{\prime}\right)=\delta\left(x-x^{\prime}\right) \delta\left(y-y^{\prime}\right) \quad \text { (Orthonormality) . }
$$

We may also show that

$$
\operatorname{tr}(|q(x)\rangle\langle p(y)| A)=\langle q(x)|A| p(y)\rangle .
$$

In fact, using the resolution of the identity operator, we have

$$
\begin{aligned}
(x, y \mid A)=\operatorname{tr}(|p(y)\rangle\langle q(x)| A) & =\int_{-\infty}^{+\infty} \mathrm{d} x^{\prime}\left\langle q\left(x^{\prime}\right) \mid p(y)\right\rangle\left\langle q(x)|A| q\left(x^{\prime}\right)\right\rangle \\
& =\int_{-\infty}^{+\infty} \mathrm{d} x^{\prime}\left\langle q(x)|A| q\left(x^{\prime}\right)\right\rangle\left\langle q\left(x^{\prime}\right) \mid p(y)\right\rangle \\
& =\langle q(x)|A| p(y)\rangle .
\end{aligned}
$$

Thus, we are able to prove the completeness relation

$$
\left.\int_{-\infty}^{+\infty} \int_{-\infty}^{+\infty} \mathrm{d} x \mathrm{~d} y \mid x, y\right)(x, y \mid=\stackrel{\cap}{I} .
$$


In fact (we use Eq. (3.14) below)

$$
\begin{aligned}
\int_{-\infty}^{+\infty} & \left.\int_{-\infty}^{+\infty} \mathrm{d} x \mathrm{~d} y \mid x, y\right)(x, y \mid A) \\
& =\int_{-\infty}^{+\infty} \int_{-\infty}^{+\infty} \mathrm{d} x \mathrm{~d} y(|q(x)\rangle\langle p(y)|)\langle q(x)|A| p(y)\rangle \\
& =\int_{-\infty}^{+\infty} \int_{-\infty}^{+\infty} \mathrm{d} x \mathrm{~d} y|q(x)\rangle\langle q(x)|A| p(y)\rangle\langle p(y)|=A .
\end{aligned}
$$

With these results, we can state the following important lemma:

Lemma 1. Let $\{X(\alpha)\}$ be a complete (or overcomplete) family of operators on $W$ indexed by a continuous-valued parameter $\alpha$ with measure $\mathrm{d} \mu(\alpha)$. Then

$$
\int \mathrm{d} \mu(\alpha) X^{\dagger}(\alpha) A X(\alpha)=\operatorname{tr}(A) . \stackrel{\cap}{I} \quad\left(\text { for any } A \in T_{1}^{1}(W)\right)
$$

Proof. By using the above results and by inserting judiciously the resolution of identity in $W$, we get

$$
\begin{aligned}
\int \mathrm{d} & \mu(\alpha) X^{\dagger}(\alpha) A X(\alpha) \\
= & \int \mathrm{d} \mu(\alpha) \mathrm{d} x \mathrm{~d} y \mathrm{~d} x^{\prime} \mathrm{d} y^{\prime}|p(y)\rangle\left\langle p(y)\left|X^{\dagger}(\alpha)\right| q(x)\right\rangle \\
& \quad \times\left\langle q(x)|A| q\left(x^{\prime}\right)\right\rangle\left\langle q\left(x^{\prime}\right)|X(\alpha)| p\left(y^{\prime}\right)\right\rangle\left\langle p\left(y^{\prime}\right)\right| \\
= & \left.\int \mathrm{d} \mu(\alpha) \mathrm{d} x \mathrm{~d} y \mathrm{~d} x^{\prime} \mathrm{d} y^{\prime}|p(y)\rangle\left(x^{\prime}, y^{\prime} \mid X(\alpha)\right)(X(\alpha) \mid x, y)(x)|A| q\left(x^{\prime}\right)\right\rangle\left\langle p\left(y^{\prime}\right)\right| \\
= & \int \mathrm{d} x \mathrm{~d} y \mathrm{~d} x^{\prime} \mathrm{d} y^{\prime}|p(y)\rangle\left\langle q(x)|A| q\left(x^{\prime}\right)\right\rangle\left\langle p\left(y^{\prime}\right)\right| \delta\left(x-x^{\prime}\right) \delta\left(y-y^{\prime}\right) \\
= & \left(\int \mathrm{d} x\langle q(x)|A| q(x)\rangle\right) \int \mathrm{d} y|p(y)\rangle\langle p(y)|=\operatorname{tr}(A) . I .
\end{aligned}
$$

\subsection{The $\Delta$ operator}

We may define now the intrinsic WW operator as a set of operators parametrized by the points of the classical phase plane

$$
\Delta(p, q)=2 V_{q}^{\dagger} U_{2 p} V_{q}^{\dagger} F^{2} .
$$

In fact, from Eq. (3.7), we see that the WW operator defined this way is Hermitian

$$
\Delta^{\dagger}(p, q)=\Delta(p, q) \quad \text { (hermiticity) }
$$


It also has unit trace

$$
\operatorname{tr}(\Delta(p, q))=(\Delta(p, q) \mid I)=1 \quad \text { (unit trace) }
$$

which can be computed easily in the position basis

$$
\begin{aligned}
\operatorname{tr}(\Delta(p, q)) & =2 \int_{-\infty}^{+\infty} \mathrm{d} \xi\left\langle q(\xi)\left|V_{q}^{\dagger} U_{2 p} V_{q}^{\dagger} F^{2}\right| q(\xi)\right\rangle \\
& =2 \int_{-\infty}^{+\infty} \mathrm{d} \xi\langle q(\xi-q) \mid q(q-\xi)\rangle \mathrm{e}^{2 \mathrm{i} p(q-\xi)} \\
& =2 \int_{-\infty}^{+\infty} \mathrm{d} \xi \delta(2 \xi-2 q) \mathrm{e}^{2 \mathrm{i} p(q-\xi)}=1 .
\end{aligned}
$$

The set $\{\Delta(p, q) / \sqrt{2 \pi}\}$ can also be shown to be orthonormal

$$
\left(\Delta(p, q) \mid \Delta\left(p^{\prime}, q^{\prime}\right)\right)=2 \pi \delta\left(p-p^{\prime}\right) \delta\left(q-q^{\prime}\right) \quad \text { (orthonormality) . }
$$

In fact, the relation above can be proved by using the next two equations. The first identity is the product formula for the WW operators

$$
\Delta(V) \cdot \Delta\left(V^{\prime}\right)=2 \Delta\left(V-V^{\prime}\right) F^{2} \mathrm{e}^{2 \mathrm{i} \Omega\left(V, V^{\prime}\right)} \quad \text { (product formula), }
$$

where

$$
\Omega\left(V, V^{\prime}\right)=p^{\prime} q-p q^{\prime}
$$

is the symplectic area spanned by the phase space vectors $V=\left(\begin{array}{c}p \\ q\end{array}\right)$ and $V^{\prime}=\left(\begin{array}{c}p^{\prime} \\ q^{\prime}\end{array}\right)$. This formula can be easily seen to hold by using Eqs. (3.5) and (3.7). The second one is the "squared Fourier transform" of the WW operator

$$
\operatorname{tr}\left(\Delta(p, q) F^{2}\right)=\pi \delta(p) \delta(q),
$$

which may again be computed in the position basis

$$
\begin{aligned}
\operatorname{tr}\left(\Delta(p, q) F^{2}\right) & =\int_{-\infty}^{+\infty} \mathrm{d} \xi\left\langle q(\xi)\left|\Delta(p, q) F^{2}\right| q(\xi)\right\rangle \\
& =2 \int_{-\infty}^{+\infty} \mathrm{d} \xi\left\langle q(\xi)\left|V_{q}^{\dagger} U_{2 p} V_{q}^{\dagger}\right| q(\xi)\right\rangle \\
& =2 \int_{-\infty}^{+\infty} \mathrm{d} \xi\left\langle q(\xi-q)\left|U_{2 p}\right| q(\xi+q)\right\rangle \\
& =\delta(q) \int_{-\infty}^{+\infty} \mathrm{d} \xi \mathrm{e}^{2 \mathrm{i} p \xi}=\pi \delta(p) \delta(q) .
\end{aligned}
$$

We used above the well-known integral representation for the Dirac "delta function"

$$
\delta(x)=\frac{1}{2 \pi} \int_{-\infty}^{+\infty} \mathrm{d} \xi \mathrm{e}^{\mathrm{i} x \xi} .
$$


The completeness relation is

$$
\int_{-\infty}^{+\infty} \int_{-\infty}^{+\infty} \frac{\mathrm{d} p \mathrm{~d} q}{2 \pi} \Delta(p, q) A \Delta(p, q)=\operatorname{tr}(A) I \quad\left(\text { for all } A \in T_{1}^{1}(W)\right) .
$$

In fact, in coordinate representation

$$
\begin{aligned}
& \int_{-\infty}^{+\infty} \int_{-\infty}^{+\infty} \frac{\mathrm{d} x \mathrm{~d} y}{2 \pi}\langle q(\xi)|\Delta(x, y) A \Delta(x, y)| q(\eta)\rangle \\
& =\frac{2}{\pi} \int_{-\infty}^{+\infty} \int_{-\infty}^{+\infty} \mathrm{d} x \mathrm{~d} y\langle q(2 y-\xi)|A| q(2 y-\eta)\rangle \mathrm{e}^{2 \mathrm{i} x(\xi-\eta)} \\
& =\delta(\xi-\eta) \int_{-\infty}^{+\infty} \mathrm{d} y\langle q(y-\xi)|A| q(y-\eta)\rangle \\
& =\delta(\xi-\eta) \int_{-\infty}^{+\infty} \mathrm{d} y\langle q(y)|A| q(y)\rangle=\delta(\xi-\eta) \operatorname{tr}(A)
\end{aligned}
$$

As we saw in the preceding subsection, we can write the above completeness relation as

$$
\left.\int \mathrm{d} \mu(V) \mid \Delta(V)\right)\left(\Delta(V) \mid=\stackrel{\cap}{I} \quad \text { with } \mathrm{d} \mu(V)=\frac{\mathrm{d} p \mathrm{~d} q}{2 \pi} .\right.
$$

Some other (not so well known) identities of easy verification from the intrinsic definition (Eq. (3.17)) are

$$
\begin{aligned}
& \Delta^{2}(V)=4 I, \\
& F^{2} \Delta(V) F^{2}=\Delta(-V), \\
& \Delta(0)=2 F^{2} .
\end{aligned}
$$

At this point, it should be worthy to stress that the WW operator defined in Eq. (3.17) is completely equivalent to the usual formalism. In fact, by inserting a convenient identity operator we obtain

$$
\begin{aligned}
\Delta(x, y) & =\int_{-\infty}^{+\infty} \mathrm{d} \xi V_{y}^{\dagger} U_{2 x}|q(\xi / 2)\rangle\langle q(\xi / 2)| V_{y}^{\dagger} F^{2} \\
& =\int_{-\infty}^{+\infty} \mathrm{d} \xi \mathrm{e}^{\mathrm{i} x \xi}|q(y+\xi / 2)\rangle\langle q(Y-\xi / 2)|
\end{aligned}
$$

which is one of the definitions for the $\Delta$ operator commonly found in literature (see Ref. [1] for instance).

At this point, we may introduce the WW transform of an arbitrary operator $A$

$$
\omega\{A\}(p, q)=(\Delta(p, q) \mid A)=a(p, q) .
$$

As one can see, the WW transform of an arbitrary operator is just the component of the operator as projected over the $\Delta$-basis, so it is, in general, a complex function on phase space. But the transform of an Hermitian operator is a real function on 
the phase plane. Classical observables are real functions defined on phase space, like energy and momentum for instance, while quantum observables are represented by Hermitian operators on Hilbert Space. Thus, one may say that quantum observables are mapped to classical-like observables. We should not take this interpretation too literally, though. This mapping is one-to-one, and so these classical functions do preserve all the quantum mechanical information of the Hermitian operators, and in this way, they are not expected to respect all the usual classical properties. For instance, it is well known that the transform of a quantum mechanical density matrix is not, in general, a positive-real-valued function, though its analogous classical density function is, of course, a positive probability distribution over the space of classical states. However, the projections of the WW transform of the density matrix corresponds to positive-definite probability distributions in position or momentum and the Husimi transform, which is a smoothing of the WW transform, is also a positive-definite function on phase space [11-13].

The WW transform can be easily inverted

$$
\left.\left.\mid A)=\int \mathrm{d} \mu(V) \mid \Delta(V)\right)(\Delta(V) \mid A)=\int \mathrm{d} \mu(V) \mid \Delta(V)\right) a(V)
$$

and the Hermitian inner product can be written in a "phase space" representation:

$$
(A \mid B)=\int \mathrm{d} \mu(V)(A \mid \Delta(V))(\Delta(V) \mid B)=\int \mathrm{d} \mu(V) a(\bar{V}) b(V),
$$

where $a(\bar{V})$ means the complex conjugate of $a(V)$. Probably, the most important (and well known) property of the WW transform is the one stated in the theorem below (and proved in the appendix) which implies that (in the $\hbar \rightarrow 0$ limit) the quantum commutator goes to the correct corresponding classical Poisson bracket.

Theorem 2. Let $A$ and $B$ be two well-defined operators in $T_{1}^{1}(W)$. Then (exhibiting explicitly Planck's constant $\hbar$ ) the transform of their product is given by

$$
(\Delta(p, q) \mid A . B)=a(p, q) \mathrm{e}^{\mathrm{i} \hbar / 2((\overleftarrow{\partial} / \partial p)(\vec{\partial} / \partial q)-(\overleftarrow{\partial} / \partial q)(\vec{\partial} / \partial p))} b(p, q)
$$

(The arrows over the derivative operators indicate which of the phase space functions they operate on.)

This, in fact, implies immediately that

$$
\hbar \stackrel{\operatorname{Lim}}{\rightarrow}\left(\frac{-\mathrm{i}}{\hbar}\right) \cdot(\Delta(p, q) \mid[A, B])=\{a(p, q), b(p, q)\} .
$$

\section{The WW transform for finite-dimensional spaces}

\subsection{Schwinger's finite-dimensional quantum kinematics}

(The main references for this subsection are Refs. $[4,16,10]$.)

The subject of "finite quantum mechanics" was widely developed by Schwinger in the 1960s [10], though pioneered by Weyl in the early days of quantum theory [9]. 
It is, in many ways, a very close analog of the usual quantum mechanics of infinitedimensional state spaces spanned by continuous-indexed basis like the position and momentum eigenvectors. In this subsection, we review briefly this formalism, mostly to standardize notation and to push the analogies as far as possible.

Let $W^{(N)}$ be an $N$-dimensional quantum space spanned by an orthonormal basis $\left\{\left|u_{k}\right\rangle\right\},(k=0,1,2, \ldots N-1)$, i.e., (We use from now on, the sum over repeated index convention, unless we explicitly mention the contrary.)

$$
\left|u_{k}\right\rangle\left\langle u^{k}\right|=I \quad \text { and } \quad\left\langle u^{j} \mid u_{k}\right\rangle=\delta_{k}^{j} .
$$

This basis plays the role of a finite set of "position states", where the position eigenvalues take values in the finite ring $Z_{N}=\{0,1,2, \ldots N-1\}$. We may introduce the unitary translation operator that acts cyclically upon these states

$$
V\left|u_{k}\right\rangle=\left|u_{k-1}\right\rangle \text {. }
$$

This means that $V^{N}=I$, so that its eigenvalues must be the $N$ th roots of unity

$$
V\left|v_{k}\right\rangle=v_{k}\left|v_{k}\right\rangle \quad \text { with } v_{k}=\mathrm{e}^{2 \pi \mathrm{i} k / N} .
$$

It can be shown that the $\left\{\left|v_{k}\right\rangle\right\}$ eigenstates also form an orthogonal basis (which we shall interpret as the discrete "momentum states"):

$$
\left|v_{k}\right\rangle\left\langle v^{k}\right|=I \quad \text { and } \quad\left\langle v^{j} \mid v_{k}\right\rangle=\delta_{k}^{j} .
$$

It can also be shown that the overlap between both basis is given by

$$
\left\langle u^{j} \mid v_{k}\right\rangle=\frac{1}{N} v_{k}^{j},
$$

where the above finite symmetric matrix is

$$
v_{k}^{j}=\left(v_{k}\right)^{j}=\mathrm{e}^{2 \pi \mathrm{i} k j / N} .
$$

Note that, in the above equation, the upper index plays a double role. It represents at the same time a contravariant index of the $v_{k}^{j}$ matrix and it also represents the $j$ th power of the $v_{k}$ eigenvalue. We will be using this kind of notation from now on.

We can define a translation operator for the momentum states as we did with the $\left\{\left|u_{k}\right\rangle\right\}$ position basis

$$
U\left|v_{k}\right\rangle=\left|v_{k+1}\right\rangle \text {. }
$$

It happens that the original position basis turns out to be the eigenstates of $U$ with the same spectrum of the momentum states

$$
U\left|u_{k}\right\rangle=v_{k}\left|u_{k}\right\rangle \quad \text { with } v_{k}=\mathrm{e}^{2 \pi \mathrm{i} k / N} .
$$

By applying different powers of the translators over one of the above basis they are easily seen to obey the well-known Weyl commutation relations

$$
V^{k} U^{l}=v^{k l} U^{l} V^{k} .
$$

We can now define the finite Fourier transform (FFT)

$$
F=\left|v_{k}\right\rangle\left\langle u^{k}\right|
$$


which is a unitary operator that takes the position basis $\left\{\left|u_{k}\right\rangle\right\}$ to the momentum basis $\left\{\left|v_{k}\right\rangle\right\}$ :

$$
F\left|u_{j}\right\rangle=\left|v_{j}\right\rangle \quad \text { and } \quad F\left|v_{j}\right\rangle=\left|u_{-j}\right\rangle .
$$

Its matrix elements are surprisingly the same in both bases:

$$
F_{j}^{k}=\left\langle u^{k}|F| u_{j}\right\rangle=\left\langle v^{k}|F| v_{j}\right\rangle=\frac{1}{\sqrt{N}} v_{j}^{k} .
$$

The unitarity of $F$ means that

$$
v_{-k}^{j} v_{l}^{k}=N \delta_{l}^{j}
$$

(remember that the index set is the finite Ring $Z_{N}$, so that $-k$ is the same as $N-k$ ).

In a perfectly analogous way with the continuous case, the FFT obeys the following properties (Sections 2 and 3):

$$
\begin{aligned}
& F^{\dagger}\left|u_{j}\right\rangle=\left|v_{-j}\right\rangle \quad \text { and } \quad F^{\dagger}\left|v_{j}\right\rangle=\left|u_{j}\right\rangle, \\
& F^{2}\left|u_{j}\right\rangle=\left|u_{-j}\right\rangle \quad \text { and } \quad F^{2}\left|v_{j}\right\rangle=\left|v_{-j}\right\rangle, \\
& F^{3}=F^{\dagger} \quad \text { and } \quad F^{4}=I
\end{aligned}
$$

and also

$$
\begin{aligned}
& F^{\dagger} U F=V^{\dagger} \quad \text { and } \quad F^{\dagger} V F=U^{\dagger}, \\
& F^{2} V F^{2}=V^{\dagger} \quad \text { and } \quad F^{2} U F^{2}=U^{\dagger} .
\end{aligned}
$$

We can introduce in the same way we did in Section 3.2, a Dirac notation for the space of operators in $W$. We define the inner product in $T_{1}^{1}\left(W^{(N)}\right)$ together with its natural operator algebra structure

$$
(A \mid B)=\operatorname{tr}\left(A^{\dagger} B\right)
$$

with $A \equiv \mid A)$ and $B \equiv \mid B)$ elements of $T_{1}^{1}\left(W^{(N)}\right)$.

$$
\mid A) \cdot(B)=\mid A B) \text {. }
$$

We can also identify, in the same way, elements of $T_{1}^{1}\left(W^{(N)}\right)$ with $T_{1}^{1}\left(T_{1}^{1}\left(W^{(N)}\right)\right)$ by the linear injective inclusion map

$$
\begin{aligned}
& i: T_{1}^{1}(W) \rightarrow T_{1}^{1}\left(T_{1}^{1}(W)\right) \\
& A \mapsto \hat{A}
\end{aligned}
$$

such that

$$
\stackrel{\cap}{A} \mid B)=\mid A B) \text {. }
$$

Let $\left.\left\{X_{\alpha} \equiv \mid X_{\alpha}\right)\right\}$ be a set of operators indexed by a finite set of $N^{2}$ elements, i.e., the dimension of $T_{1}^{1}\left(T_{1}^{1}(W)\right)$. The set is complete and orthonormal if

$$
\left.\mid X_{\alpha}\right)\left(X^{\alpha}|=I=| I\right)=\stackrel{\cap}{I}
$$

and

$$
\left(X^{\alpha} \mid X_{\beta}\right)=\operatorname{tr}\left(X_{\alpha}^{\dagger} X_{\beta}\right)=\delta_{\beta}^{\alpha}, \quad \text { where } X^{\alpha}=X_{\alpha}^{\dagger} .
$$


Let us define the following $N^{2}$ operators

$$
\left|u_{j}\right\rangle\left\langle v^{k}|=| \begin{array}{l}
k \\
j
\end{array}\right\rangle \text {. }
$$

They form clearly a complete and orthonormal set

$$
\begin{aligned}
& \left(\begin{array}{c}
m \\
n
\end{array} \mid \begin{array}{l}
k \\
j
\end{array}\right)=\delta_{j}^{m} \delta_{n}^{k}, \\
& \left.\mid \begin{array}{l}
k \\
j
\end{array}\right)\left(\begin{array}{l}
j \\
k
\end{array} \mid=I .\right.
\end{aligned}
$$

In fact, we have

$$
\left(\left.\begin{array}{c}
m \\
n
\end{array}\right|_{j} ^{k}\right)=\operatorname{tr}\left(\left|v_{n}\right\rangle\left\langle u^{m} \mid u_{j}\right\rangle\left\langle v^{k}\right|\right)=\left\langle u^{m} \mid u_{j}\right\rangle\left\langle v^{k} \mid v_{n}\right\rangle=\delta_{j}^{m} \delta_{n}^{k} .
$$

To prove completeness, we will consider first the following result. For all $A \in T_{1}^{1}(W)$, we have

$$
\left(\begin{array}{c}
m \\
n
\end{array} \mid A\right)=\left\langle u^{m}|A| v_{n}\right\rangle
$$

which can be computed easily

$$
\left(\begin{array}{c}
m \\
n
\end{array} \mid A\right)=\operatorname{tr}\left\{\left(\left|u_{m}\right\rangle\left\langle v^{n}\right|\right)^{\dagger} A\right\}=\operatorname{tr}\left\{\left|v_{n}\right\rangle\left\langle u^{m}\right| A\right\}=\left\langle u^{m}|A| v_{n}\right\rangle,
$$

so that indeed

$$
\left.\mid \begin{array}{l}
k \\
j
\end{array}\right)\left(\begin{array}{l}
j \\
k
\end{array} \mid A\right)=\left(\left|u_{j}\right\rangle\left\langle v^{k}\right|\right)\left\langle u^{j}|A| v_{k}\right\rangle=\left|u_{j}\right\rangle\left\langle u^{j}|A| v_{k}\right\rangle\left\langle v^{k}\right|=A .
$$

If $\left\{X_{\alpha}\right\}$ is a complete set of operators, then

$$
X^{\alpha} A X_{\alpha}=\operatorname{tr}(A) I \quad(\text { sum over } \alpha) .
$$

In fact,

$$
\begin{aligned}
X^{\alpha} A X_{\alpha} & =\left|v_{j}\right\rangle\left\langle v^{j}\left|X^{\alpha}\right| u_{k}\right\rangle\left\langle u^{k}|A| u_{m}\right\rangle\left\langle u^{m}\left|X_{\alpha}\right| v_{r}\right\rangle\left\langle v^{r}\right| \\
& =\left|v_{j}\right\rangle\left(\left.X^{\alpha}\right|_{k} ^{j}\right)\left\langle u^{k}|A| u_{m}\right\rangle\left({ }_{r}^{m} \mid X_{\alpha}\right)\left\langle v^{r}\right| \\
& =\left|v_{j}\right\rangle\left({ }_{r}^{m} \mid X_{\alpha}\right)\left(\left.X^{\alpha}\right|_{k} ^{j}\right)\left\langle v^{r}\right|\left\langle u^{k}|A| u_{m}\right\rangle \\
& =\left|v_{j}\right\rangle \delta_{k}^{m} \delta_{r}^{j}\left\langle v^{r}\left|\left\langle u^{k}|A| u_{m}\right\rangle=\right| v_{r}\right\rangle\left\langle v^{r}\right|\left\langle u^{m}|A| u_{m}\right\rangle=\operatorname{tr}(A) I .
\end{aligned}
$$

\subsection{The finite $W W$ basis}

\subsubsection{Odd-dimensional spaces}

Suppose now that the dimension of $W^{(N)}$ is an odd number. Define $N^{2}$ operators in $T_{1}^{1}(W)$ as

$$
\Delta_{m n}=V^{-n} U^{m} V^{-n} F^{2} \quad\left(m, n \in Z_{N}\right) .
$$

Notice the absence of the factor 2 in comparison with its continuous analog.

Using Eqs. (4.9) and (4.15), one can show that these operators are Hermitian:

$$
\Delta_{m n}^{\dagger}=\Delta^{m n}=\Delta_{m n} \text {. }
$$

They are also of unitary trace:

$$
\operatorname{tr}\left(\Delta_{m n}\right)=\left(I \mid \Delta_{m n}\right)=1 .
$$


In fact,

$$
\begin{aligned}
\left\langle u^{j}\left|\Delta_{m n}\right| u_{j}\right\rangle & =\left\langle u^{j}\left|V^{-n} U^{m} V^{-n} F^{2}\right| u_{j}\right\rangle \\
& =\left\langle u^{j-n}\left|U^{2 m}\right| u_{n-j}\right\rangle=v_{n-j}^{2 m} \delta_{n-j}^{j-n}=v_{n-j}^{2 m} \delta^{j n}=v_{0}^{2 m}=1 .
\end{aligned}
$$

Note that it makes since to "invert" the element $2 \in Z_{N}$, between $N$ is odd. In fact, using the "Fermat-Euler theorem", we have explicitly $[4,10]$

$$
2^{-1}=2^{\varphi(N)-1}(\operatorname{Mod} N),
$$

where $\varphi(N)$ is the so-called Euler's totient number-theoretical function. It maps the positive integers $N$ to the number of non-divisors of $N$. In fact, for even $N$, the above definition would not lead, in a consistent way, to a set of $N^{2}$ linear independent operators. (Take $m=N / 2$ for instance.) One can see from the definition that

$$
\Delta_{m n}^{2}=I
$$

which leads to the completeness of the set $\left\{\Delta_{m n} / \sqrt{N}\right\}$

$$
\left.\frac{1}{N} \mid \Delta_{m n}\right)\left(\Delta^{m n}|=| I\right)(I \mid \equiv I \text {. }
$$

In fact, from the cycle property of trace, we have for all operators $A$

$$
\operatorname{tr}\left(\frac{1}{\sqrt{N}} \Delta_{m n} \cdot A \cdot \frac{1}{\sqrt{N}} \Delta^{m n}\right)=\frac{1}{N} \sum_{m, n} \operatorname{tr}(A)=N \operatorname{tr}(A)=(\operatorname{tr} I) \operatorname{tr}(A) .
$$

By taking the inner product of $\mid I$ ) with Eq. (4.31) and using Eq. (4.28), we may derive the following expansion for the identity operator:

$$
I=\frac{1}{N} \sum_{m, n} \Delta_{m n}
$$

Let us define now the finite WW transform of an arbitrary operator $A$ as

$$
\varpi^{m n}[A]=\left(\Delta^{m n} \mid A\right)=\operatorname{tr}\left(\Delta^{m n} A\right)=a^{m n}
$$

which can be inverted

$$
\left.A=\frac{1}{N} \mid \Delta_{m n}\right)\left(\Delta^{m n} \mid A\right)=\frac{1}{N} \Delta_{m n} a^{m n} .
$$

We can easily compute the inner product of two operators $A$ and $B$ if we know their transforms $a^{m n}$ and $b^{m n}$ :

$$
(A \mid B)=\frac{1}{N}\left(A \mid \Delta_{m n}\right)\left(\Delta^{m n} \mid B\right)=\frac{1}{N} a_{m n} b^{m n} .
$$

(Notice that $a^{m n}=\bar{a}_{m n}$ in general, but for any Hermitian operator $A$, we have $a_{m n}=$ $\bar{a}_{m n}$.)

Introducing a "vector notation" for finite phase space (as we did for the continuous case) we can write the product of two $\Delta$ operators (using (4.9) and (4.15):

$$
\Delta_{\vec{x}} \Delta_{\vec{x}^{\prime}}=v_{2 \Omega\left(\vec{x}, \vec{x}^{\prime}\right)} \Delta_{\vec{x}-\vec{x}^{\prime}} F^{2},
$$


where

$$
\vec{x}=\left(\begin{array}{l}
m \\
n
\end{array}\right) \quad \text { and } \quad \vec{x}^{\prime}=\left(\begin{array}{c}
m \\
n^{\prime}
\end{array}\right)
$$

and the finite "symplectic area" is

$$
\Omega\left(\vec{x}, \vec{x}^{\prime}\right)=n m^{\prime}-n^{\prime} m .
$$

The finite squared Fourier transform of the $\Delta$ operator can be found by the product at the right of Eq. (4.32) (by $\left.\Delta_{p q} F^{2}\right)$ together with some index manipulation

$$
\Delta_{p q} F^{2}=\frac{1}{N} \Delta_{m n} v_{q}^{-2 m} v_{p}^{2 n} .
$$

The $N^{2} \Delta_{p q}$ operators form clearly a complete set of orthonormal generators for the complexified algebra $u(N)$ of the Lie-group of unitary transformations of $W^{(N)}$ (for odd $N$ ).

The structure constants of $U(N)$ may then be computed in this basis. In fact, using again the vector notation we arrive at the following commutation relations (see also Ref. [7]):

$$
\left[\Delta_{\vec{x}}, \Delta_{\vec{y}}\right]=\frac{2 i}{N} \Delta_{\vec{z}} \Lambda_{\vec{x} \vec{y}}^{\vec{z}} .
$$

The sum here is over the "collective" index $\vec{z}$, and the structure constants $\Lambda_{\vec{x} \vec{y}}^{\vec{z}}$ are given explicitly by

$$
\Lambda_{\vec{x} \vec{y}}^{\vec{z}}=\sin \left[\left(\frac{4 \pi}{N}\right)(\Omega(\vec{x}, \vec{y})+\Omega(\vec{y}, \vec{z})+\Omega(\vec{z}, \vec{x}))\right] .
$$

\subsubsection{Even-dimensional spaces}

We can partially extend this formalism to even dimensional spaces in the following form.

Let us start defining $N^{2}$ unitary operators over $W^{(N)}$ in the following way (This procedure can be carried out both for even and odd dimensional spaces, but it will be equivalent to the preceding discussion for odd $N$.):

$$
X_{m n}=U^{m} V^{n} .
$$

We redefine these operators by a phase

$$
S_{m n}=X_{m n} v_{m n / 2} \text {. }
$$

In the complex phase $v_{m n / 2}$, the index $m n / 2$ means the $Z_{N}$ elements $2^{-1} m n$ if $N$ is odd. This is because, for odd $N, Z_{N}$ is a finite field and any element of $Z_{N}$ has an inverse [14]. However, for even $N$, we establish the following convention. The complex phase $v_{m n / 2}$ will be defined uniquely by the exponential $\mathrm{e}^{\pi \mathrm{i} m n / N}$ where $m$ and $n$ are the unique representatives of the $Z_{N}$ ring between 0 and $N$. This must be done because the above exponential is clearly not a $\bmod N$ invariant. In this way, the $S_{m n}$ operators are well defined for both even and odd-dimensional spaces. From Eq. (4.9) we see that

$$
X_{m n}^{\dagger}=v_{m n} X_{-m-n}
$$


so that

$$
S_{m n}^{\dagger}=S_{-m-n} .
$$

Let us compute the trace of $X_{m n}$ explicitly:

$$
\operatorname{tr}\left(X_{m n}\right)=\left\langle u^{j}\left|U_{m} V_{n}\right| u_{j}\right\rangle=\left\langle u^{j} \mid u_{j-n}\right\rangle v^{m j}=\delta_{j-n}^{j} v^{m j} .
$$

The above equation is null for all values of $m$ and $n$ unless $m=n=0$, when the sum equals $N$. So we have

$$
\operatorname{tr}\left(X_{m n}\right)=\operatorname{tr}\left(S_{m n}\right)=\left(I \mid X_{m n}\right)=N \delta_{m}^{0} \delta_{n}^{0} .
$$

Now, we can define the $\Delta$ operators as

$$
\Delta_{m n}=\frac{1}{N} S_{j k} v_{m}^{-j} v_{n}^{-k} \text {. }
$$

Using Eqs. (4.19) and (4.20), it is possible to see that indeed the above definition gives us a set of Hermitian and unit trace operators. For odd dimension we can prove the equivalence of both approaches. In fact, for odd $N$, we can write

$$
\Delta_{m n}=S_{2 m-2 n} F^{2} \quad(\text { odd } N) .
$$

Because of Eqs. (4.32) and (4.36) we may write

$$
\Delta_{p q}=\frac{1}{N} \sum_{m, n} \Delta_{m n} \Delta_{p q}=\frac{1}{N} \Delta_{m-p n-q} F^{2} v_{p}^{2 n} v_{q}^{-2 m} .
$$

Making the substitutions $m-p=j$ and $n-q=k$ in the above equation and also using Eq. (4.47), we arrive at Eq. (4.46). If $N$ is even, the completeness and orthonormality of $\left\{(1 / \sqrt{N}) \Delta_{m n}\right\}$ follows from the completeness and orthonormality of the bases $\left\{(1 / \sqrt{N}) X_{m n}\right\}$ and $\left\{(1 / \sqrt{N}) S_{m n}\right\}$. In fact, note first that (by Eq. (4.9))

$$
X_{m n} X_{p q}=v_{n p} X_{m+p n+q}
$$

which leads to

$$
\left(X^{m n} \mid X_{p q}\right)=\operatorname{tr}\left(X_{-m-n} X_{p q}\right) v_{m n}=\operatorname{tr}\left(X_{p-m} q-n\right) v_{n(m-p)}=N v_{n(m-p)} \delta_{p-m}^{0} \delta_{q-n}^{0}
$$

which implies

$$
\left(X^{m n} \mid X_{p q}\right)=\left(S^{m n} \mid S_{p q}\right)=N \delta_{p}^{m} \delta_{q}^{n} .
$$

In a similar form, the completeness of $\left\{(1 / \sqrt{N}) X_{m n}\right\}$ and $\left\{(1 / \sqrt{N}) S_{m n}\right\}$ means that

$$
S_{m n} S^{m n}=X_{m n} X^{m n}=N^{2} I .
$$

In fact, we have

$$
S_{m n} S^{m n}=X_{m n} X^{m n}=\sum_{m, n} U^{m} V^{n} V^{-n} U^{-m}=I \sum_{m, n}=N^{2} I
$$

from where we can derive by a straightforward computation the completeness for the $\Delta$ operators:

$$
\Delta_{p q} \Delta^{p q}=N^{2} I
$$


The unit trace property follows directly from Eq. (4.45), so these are all common properties both for even- and odd-dimensional spaces. On the other hand, the fact that the square of the $\Delta$ operator is the identity operator (Eq. (4.30)) is shared only by the odd-dimensional case. As a counterexample, let us take $N=2$. In the $\left\{\left|u_{0}\right\rangle,\left|u_{1}\right\rangle\right\}$ basis, we may compute explicitly

$$
\begin{aligned}
& \Delta_{00}=\frac{1}{2}\left(\begin{array}{cc}
2 & -1-i \\
1-i & 0
\end{array}\right), \quad \Delta_{01}=\frac{1}{2}\left(\begin{array}{cc}
0 & 1-i \\
1+i & 2
\end{array}\right), \\
& \Delta_{10}=\frac{1}{2}\left(\begin{array}{cc}
2 & 1+i \\
-1+i & 0
\end{array}\right), \quad \Delta_{11}=\frac{1}{2}\left(\begin{array}{cc}
0 & -1+i \\
-1-i & 2
\end{array}\right) .
\end{aligned}
$$

The square of these operators are not even proportional to the identity operator as can be easily verified. From an algebraic point of view, this is an advantage for odd-dimensional spaces. For odd $N$, we can exponentiate the $\Delta$ operators in a simple form:

$$
\mathrm{e}^{\mathrm{i} \theta \Delta}=(\cos \theta) I+\mathrm{i}(\sin \theta) \Delta \quad(\operatorname{odd} N) .
$$

Unfortunately, there is no such simple similar equation for even-dimensional spaces.

\section{Closing remarks}

We have carried out a brief review for the Weyl-Wigner (WW) transform theory and presented it in a slightly different manner than usual. To accomplish this, we have introduced first, an intrinsic definition of the Fourier transform operator making it possible to exhibit a "coordinate-free" formula for the WW $\Delta$ operator basis itself. This permits us to (besides greatly simplifying the whole formalism) show some new algebraic identities related to the $\Delta$ operators. A coordinate-independent formulation of the WW representation has also been presented in Ref. [13]. The author there delivers an extensive and deep account of the WW representation in a geometrical semi-classical perspective where the translation and reflection operators in phase space are introduced in an algebraic structure which is largely explored to construct the WW representation leading to a path integral formulation of the Weyl propagator. We believe that our approach is also a very good frame to study a finite dimensional system generalization of these "classical-like" mathematical structures of quantum phase space. In fact, in the finite case, we have seen some striking differences between the structure of these systems depending on whether the underlying quantum vector space is of even or odd dimension. Some other kind of "anomalies" for even-dimensional spaces have been reported by us before [6] (see also Ref. [15]). Here also we must point out Ref. [16] where the authors have also translated the WW theory to finite dimensional systems where the distinction of even- and odd-dimensional spaces is analyzed in terms of the geometric support of the reflection operators. It is our intention to return to this specific subject in a future work [17].

It is interesting to note that, for finite spaces, the $\Delta$ operators form a special basis of generators of the $u(N)$ algebra. Following this idea, one may try to re-interpret the $\Delta$-basis for the usual continuous infinite dimensional theory as a set of generators 
for some kind of a " $u(\infty)$ algebra". These formula for the finite WW operators have also been derived in Ref. [15] in a quite different approach. In a certain sense, one may say that our procedure is the inverse of theirs. Here, we have started with a definition that is a direct analog of the continuous infinite-dimensional case, which we developed in Sections 2 and 3. There, the authors work with a angle-angular momentum interpretation. They have started with a list of properties that they expected to be a sensible definition for a finite-dimensional WW operator and they have shown that it leads to a WW operator that is equivalent to the one we have just presented. Besides all this, the intrinsic formula for the WW $\Delta$ operators is also essential to build a $q$-deformed generalization of WW formalism that we report elsewhere [18].

\section{Acknowledgements}

A.C.L. and M.C.N. are partially supported by Conselho Nacional de Desenvolvimento Científico e Tecnológico, CNPq. The authors wish to thank the referee of Physica A for valuable suggestions and for bringing to our attention Refs. [11-13,16]. We also appreciate the critical reading of the manuscript by D. Galetti from the Instituto de Física Teórica-Unesp.

\section{Appendix A. Proof of Theorem 2}

Theorem 2 states a well-known formula for the WW transform of the product of two arbitrary operators:

$$
(\Delta(p, q) \mid A \cdot B)=a(p, q) \mathrm{e}^{\mathrm{i} / 2((\overleftarrow{\partial} / \partial p)(\vec{\partial} / \partial q)-(\overleftarrow{\partial} / \partial q)(\vec{\partial} / \partial p))} b(p, q) \quad(\hbar=1) .
$$

We present a proof using the generalized "bra-ket" language that we introduced in Section 3 (see also Ref. [1]). Let us consider first, two additional lemmas

Lemma A.1. The identity operator in $W$ may be expanded in the $\Delta$ basis as

$$
I=\int \mathrm{d} \mu\left(V^{\prime}\right) \Delta\left(V^{\prime}\right)
$$

where $\mathrm{d} \mu(V)=(1 / 2 \pi) \mathrm{d} p \mathrm{~d} q$ and $V=\left(\begin{array}{c}p \\ q\end{array}\right)$.

Lemma A.2. The squared Fourier transform of the $\Delta$ operator is

$$
\Delta(V) F^{2}=2 \int \mathrm{d} \mu\left(V^{\prime}\right) \Delta\left(V^{\prime}\right) \mathrm{e}^{-2 \mathrm{i} \Omega\left(V, V^{\prime}\right)},
$$

where $\Omega\left(V, V^{\prime}\right)=p^{\prime} q-p q^{\prime}$.

Lemma 3 is a consequence of the following computation:

$$
\left.\left.\mid I)=\int \mathrm{d} \mu(V) \mid \Delta(V)\right)(\Delta(V) \mid I)=\int \mathrm{d} \mu(V) \mid \Delta(V)\right)
$$


and Lemma A.2 follows from Lemma A.1 by the product of Eq. (A.1) by $\Delta(V) F^{2}$ (together with Eq. (3.21)).

$$
\Delta(V) F^{2}=\int \mathrm{d} \mu\left(V^{\prime}\right) \Delta\left(V^{\prime}\right) \Delta(V) F^{2}=2 \int \mathrm{d} \mu\left(V^{\prime}\right) \Delta\left(V^{\prime}-V\right) F^{2} \mathrm{e}^{-2 \mathrm{i} \Omega\left(V^{\prime}, V^{\prime}\right)} .
$$

Making the translation $V^{\prime} \rightarrow V+V^{\prime}$ in the above equation, and noting that the measure is invariant under translations in phase space, one arrives at Eq. (A.2).

With this, we may now compute the transform of a product of operators:

$$
\begin{aligned}
(\Delta(V) \mid A . B)=(\Delta(V)|\hat{A}| B) & =\int \mathrm{d} \mu\left(V^{\prime}\right)\left(\Delta(V)|\hat{A}| \Delta\left(V^{\prime}\right)\right)\left(\Delta\left(V^{\prime}\right) \mid B\right) \\
& =\int \mathrm{d} \mu\left(V^{\prime}\right)\left(\Delta(V) \Delta\left(V^{\prime}\right) \mid A\right) b\left(V^{\prime}\right) \\
& =2 \int \mathrm{d} \mu\left(V^{\prime}\right)\left(\Delta\left(V-V^{\prime}\right) F^{2} \mid A\right) b\left(V^{\prime}\right) \mathrm{e}^{-2 \mathrm{i} \Omega\left(V, V^{\prime}\right)} .
\end{aligned}
$$

Finally, making again a translation $V^{\prime} \rightarrow V-V^{\prime}$ we find

$$
(\Delta(V) \mid A . B)=2 \int \mathrm{d} \mu\left(V^{\prime}\right)\left(\Delta\left(V^{\prime}\right) F^{2} \mid A\right) b\left(V-V^{\prime}\right) \mathrm{e}^{-2 \mathrm{i} \Omega\left(V, V^{\prime}\right)} .
$$

Now, we return to the explicit use of coordinates $V=\left(\begin{array}{c}p \\ q\end{array}\right)$ and $V^{\prime}=\left(\begin{array}{c}p^{\prime} \\ q^{\prime}\end{array}\right)$ and expand $b\left(V-V^{\prime}\right)=b\left(p-p^{\prime}, q-q^{\prime}\right)$ in a Taylor series around $V^{\prime}$ as

$$
b\left(p-p^{\prime}, q-q^{\prime}\right)=\mathrm{e}^{\left(-p \partial / \partial p-q^{\prime} \partial / \partial q\right)} b(p, q) .
$$

So we have (using lemma A.2):

$$
\begin{aligned}
(\Delta(p, q) \mid A . B) & \\
= & \frac{1}{\pi} \int_{-\infty}^{+\infty} \int_{-\infty}^{+\infty} \mathrm{d} p \mathrm{~d} q^{\prime} \mathrm{e}^{2 \mathrm{i}\left(p^{\prime} q-p q^{\prime}\right)}\left(\Delta\left(p^{\prime}, q^{\prime}\right) F^{2} \mid A\right) \mathrm{e}^{\left(-p \partial / \partial p-q^{\prime} \partial / \partial q\right)} b(p, q) \\
= & \left(\frac{1}{\pi} \int_{-\infty}^{+\infty} \int_{-\infty}^{+\infty} \mathrm{d} p^{\prime} \mathrm{d} q^{\prime} \Delta\left(p^{\prime}, q^{\prime}\right) F^{2} \mid A\right) \\
& \times \mathrm{e}^{-\mathrm{i} / 2((\overleftarrow{\partial} / \partial p)(\vec{\partial} / \partial q)-(\overleftarrow{\partial} / \partial q)(\vec{\partial} / \partial p))} b(p, q) \\
= & a(p, q) \mathrm{e}^{-\mathrm{i} / 2((\overleftarrow{\partial} / \partial p)(\vec{\partial} / \partial q)-(\overleftarrow{\partial} / \partial q)(\vec{\partial} / \partial p))} b(p, q),
\end{aligned}
$$

where the "arrows" over the differential operators in the exponential indicate which phase space function they act upon.

\section{References}

[1] S.R. de Groot, L.G. Suttorp, Foundations of Electrodynamics, North-Holland, Amsterdam, 1972 (Chapter VI). 
[2] M.J. Bastiaans, J. Opt. Soc. Amer. A 69 (1979) 1710;

T.A.C.M. Claasen, W.F.G. Mecklenbrauker, Philips J. Res. 35 217;

L.B. Almeida, IEEE Trans. Signal Process. 42 (1994) 11;

D.F. James, G.S.Z. Agarwal, Opt. Commun. 126 (1996) 207.

[3] J. Schwinger, Proc. Natl. Acad. Sci. 46 (1960) 570;

T.S. Santhanam, A.R. Tekumalla, Found. Phys. 6 (5) (1976);

P. Stovicek, J. Tolar, Rep. Math. Phys. 20 (1984);

W.K. Wooters, Ann. Phys. 176 (1987);

D. Galetti, A.F.R.T. Piza, Physica A 149 (1988);

M. Saraceno, Ann. Physics 199 (1990);

R. Aldrovandi, D. Galetti, J. Math. Phys. 31 (12) (1990);

A. Vourdas, J. Phys. A 29 (1996).

[4] A.C. Lobo, M.C. Nemes, Physica A 186 (1995) 513.

[5] A. Bohm, The rigged hilbert space and quantum mechanics, Lecture Notes in Physics, Vol. 78, Springer, New York, 1978.

[6] A.C. Lobo, M.C. Nemes, Physica A 241 (1997) 637.

[7] T. Hakioglu, 1999, lanl e-print quant-ph/9906083, available at http://xxx.lanl.gov.

[8] H.M. Ozaktas, Kutay, Introduction to the Fractional Fourier Transform and its Applications in Advances in Imaging and Electron Physics, Vol. 106, Academic Press, New York, 1999, p. 239 (Chapter 4).

[9] H. Weyl, Theory of Groups and Quantum Mechanics, Dover, New York, 1950.

[10] J. Schwinger, Quantum Kinematics and Dynamics, Benjamin, New York, 1970.

[11] K. Husimi, Proc. Phys. Math. Soc. Japan 22 (1940) 264.

[12] J. Takashi, Phys. Soc. Japan 55 (1986) 762.

[13] A.M. Ozorio de Almeida, Phys. Rep. 295 (1998) 265-342.

[14] K. Ireland, M. Rosen, A Classical Introduction to Modern Number Theory, Springer, Berlin, 1987.

[15] A. Luis, J. Perina, J. Phys. A 31 (1998) 1423.

[16] A.M.F. Rivas, A.M. Ozorio de Almeida, Ann. Phys. 276 (1999) 223-256.

[17] A.C. Lobo, Algebraic Anomalies of Even Dimensional Quantum Mechanical Systems, in preparation.

[18] A.C. Lobo, D. Galetti, in preparation. 